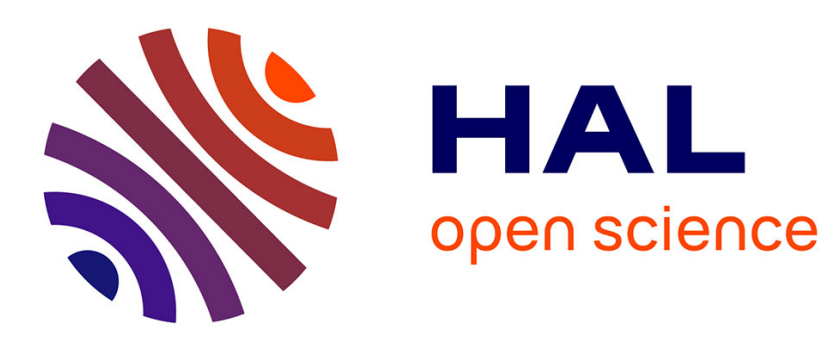

\title{
conditional sampling and scale analysis of the marine atmospheric mixed layer - SOFIA Experiment
}

\author{
Anne Réchou, Pierre Durand
}

\section{To cite this version:}

Anne Réchou, Pierre Durand. conditional sampling and scale analysis of the marine atmospheric mixed layer - SOFIA Experiment. Boundary-Layer Meteorology, 1997, 82 (01), pp.81-104. hal-02112028

\section{HAL Id: hal-02112028 \\ https://hal.univ-reunion.fr/hal-02112028}

Submitted on 26 Apr 2019

HAL is a multi-disciplinary open access archive for the deposit and dissemination of scientific research documents, whether they are published or not. The documents may come from teaching and research institutions in France or abroad, or from public or private research centers.
L'archive ouverte pluridisciplinaire HAL, est destinée au dépôt et à la diffusion de documents scientifiques de niveau recherche, publiés ou non, émanant des établissements d'enseignement et de recherche français ou étrangers, des laboratoires publics ou privés. 


\title{
CONDITIONAL SAMPLING AND SCALE ANALYSIS OF THE MARINE ATMOSPHERIC MIXED LAYER - SOFIA EXPERIMENT
}

\author{
ANNE RÉCHOU and PIERRE DURAND \\ Laboratoire d'Aérologie, UMR CNRS/UPS 5560, Observatoire Midi-Pyrénées, 14 avenue E. Belin, \\ F31400, Toulouse, France
}

(Received in final form 11 June, 1996)

\begin{abstract}
During the SOFIA experiment, performed in the Azores region in June 1992, airborne missions were conducted in the atmospheric boundary layer with two aircraft instrumented for turbulence measurements. We show how the conditional sampling technique, applied to the velocity, temperature and moisture fluctuations, is able to describe the various parcels which constitute the turbulent field. Each parcel, so identified, is characterized by its fractional area and by its contribution to the transfers of sensible heat and latent heat. On the other hand, a scale analysis is conducted by filtering the turbulent signals in five non-overlapping frequency bands, defined according to the characteristic turbulent scales. The contribution of each band to the turbulent energy and to the transfers is thus presented. The importance of the lowest frequencies, which are generally removed from the signals by high-pass filtering before computing turbulent fluxes, is shown. In the final section, the conditional sampling technique is applied to the signals filtered in the various bands. Despite a slight deformation of the eddies due to the filtering technique, the contribution of each parcel can be estimated at the various scales analysed.
\end{abstract}

\section{Introduction}

Over the past ten years, the marine atmospheric boundary layer (MABL) has received great attention from atmospheric scientists, from both a numerical and also an experimental approach. In sub-tropical areas, extensive low-level stratocumulus (Sc) clouds are frequently observed off the west coast of major continents. Over warmer water (towards the equator) fields of trade cumulus appear. Processes which intervene to maintain or break up maritime Sc layers were investigated during the ASTEX campaign, which took place in June 1992 in the Azores region (Albrecht et al., 1995). During ASTEX, the french SOFIA program was performed in the same area, with scientific objectives devoted to the study of air-sea interaction and atmospheric boundary-layer dynamics (Weill et al., 1995).

A lot of papers have recently been published dealing with the dynamical, radiative and microphysical processes in the Sc-topped MABL (see for instance the special issue of the Journal of Atmospheric Sciences (Vol. 52, August 1995) devoted to the ASTEX results). We have chosen to focus our interest on the dynamics of the low-level mixed layer, driven by surface fluxes of buoyancy and momentum. Although the fair-weather, daytime continental convective boundary layer has been extensively studied over the past thirty years, our knowledge of the mid-latitude MABL still needs to be improved.

In a preceding paper (Réchou et al., 1995, hereafter R95), we have shown from aircraft data how the turbulence moments, in the mixed layer in the SOFIA 
experiment, could be parameterized. The aim of this paper is to describe in greater detail the turbulent structure of the mixed layer, by a characterization of the various air parcels with conditional sampling techniques and appropriate filtering.

\section{General Conditions of the MABL}

The meteorological conditions encountered during SOFIA are described in R95. To summarize, the MABL is topped by a permanent trade inversion, under which develops a Sc layer. In the afternoon during which the airborne experiments were conducted, the Sc are scattered, and decoupled from the surface, i.e., the mixed layer, driven by surface fluxes, does not extend up to the Sc layer. The top of the mixed layer, $h$, is therefore much lower than the inversion altitude, $Z_{i}$. Some cumuli were sometimes present at the top of the mixed layer. The potential temperature and humidity mixing ratio are well mixed in the mixed layer driven by surface fluxes, whereas vertical gradients (positive for potential temperature and negative for humidity mixing ratio) appear in the intermediate (between mixed layer and cloud layer) layer. An illustration of such profiles is given in R95. The diurnal cycle of the Sc layer is important, with a minimum in the cloud coverage during the afternoon and a maximum at the end of the night and during the morning (Betts et al., 1995). Because of the low values of the surface heat flux (about $10 \mathrm{~W} \mathrm{~m}^{-2}$ for the sensible heat flux and $100 \mathrm{~W} \mathrm{~m}^{-2}$ for the latent heat flux), the diurnal cycle of the thermodynamical profiles in the mixed layer is weak. R95 have shown that the convective scales $w_{*}$ and $\theta_{*}$ (Deardorff, 1970) are relevant for turbulence moments parameterization, provided that $\mathrm{h}$ is used instead of $Z_{i}$ as length scale. The principal turbulence moments thus follow the convective laws established for convective continental boundary layers, at least in the lower half of the mixed layer, despite the low values of the surface buoyancy flux. Above, the behavior of the mixed layer of SOFIA significantly differs from that of the continental boundary layer, essentially because its top is not marked by an inversion and the entrainment processes are therefore weak. The surface buoyancy flux is too small to produce any significant growing of the mixed layer by encroachment, at least during the airborne experiments which lasted about two hours. As a consequence, the results presented here can be considered as representative of any convective boundary layer in its lower half but, as far as the whole mixed layer is concerned, are only valid for the marine, decoupled Sc boundary layer.

Four days were analysed, during which five flights were performed by two aircraft, a Fokker 27 (instrumented by INSU*) and a Merlin IV (operated by "Météo-France", the French Weather service). Without entering into details of the instrumentation and data processing (see R95), we only want to say here that the turbulent fluctuations in temperature, moisture and wind (three components) can be

* INSU: Institut National des Sciences de l'Univers (National Institute of the Sciences of the Universe). 
computed at scales ranging from approximately $8 \mathrm{~m}$ to $10 \mathrm{~km}$. Turbulence moments were then calculated from these time series on straight and level runs of about 25 to $30 \mathrm{~km}$ long.

Considering the horizontal homogeneity of the surface boundary conditions (sea-surface temperature does not vary significantly), and of the meteorological fields (steady anticyclone conditions), we have assumed that the turbulence characteristics only vary with altitude.

\section{Conditional Sampling of the Mixed Layer}

The conditional sampling technique has been widely used in the boundary layer, often from aircraft measurements (see for instance Lenschow and Stephens (1980; 1982) or Young (1988) for daytime convective boundary layers; Shaw and Businger (1985), Greenhut and Khalsa (1982) for low altitude MABL; Nicholls (1989); Khalsa (1993) for stratocumulus-topped MABL). It consists in selectively sampling the time series according to specified criteria. For example, various parcels can be identified according to whether their values (in temperature, moisture, velocity ... ) exceed a threshold value or not. If the threshold value is set to the ensemble average of the sample (generally zero if we consider turbulent fluctuations), then the sampling defines two classes, according to the sign of the fluctuation. If the thresholds are set to non-zero values, then three classes can be defined: greater than the upper threshold, lower than the lower threshold, and between the two. The latter class is often called "environment". This technique can be simultaneously applied to one or several parameters. So, if $n$ is the number of classes defined for each variable, and $m$ the number of variables, the total number of classes is $p=n^{m}$. In the work presented here, only one threshold has been considered and set to the average value of the sample $(n=2)$. This criterion has been successively applied to one variable $(p=2)$, two variables $(p=4)$, and to 3 variables $(p=8)$. The variables analysed in this way are the vertical velocity $w$, the potential temperature $\theta$, and the specific humidity $Q$. Each class of parcel can thus be characterized by the fractional area it occupies, and by its contribution to the transfers of heat or momentum. Before being conditionally sampled, the time series were high-pass filtered on each sample (a straight and level run of 25 to $30 \mathrm{~km}$ long) in order to remove frequencies lower than $0.024 \mathrm{~s}^{-1}$. Given the true airspeed of the aircraft, this operation removes the wavelengths greater than about $3.5 \mathrm{~km}$. Filtering is indispensable since mesoscale variability in thermodynamic fields induces lowfrequency drifts in the corresponding time series. Conditional sampling, which is used to characterize the various turbulence parcels, cannot be satisfactorily used if such tendencies are present. 


\subsection{SingLE-VARIATE CONDITIONAL SAMPLING}

Each signal is sampled according to the sign of its fluctuation. Let us consider $x$ as a turbulent signal (i.e. a signal from which the mean has been removed). If the sample of $x$ is composed of $N$ values among which $N^{+}$are positive and $N^{-}$ negative, then we have:

$$
\begin{aligned}
& N=N^{+}+N^{-}, \\
& \overline{x^{+}}=\left(\Sigma x_{i}^{+}\right) / N^{+}, \\
& \overline{x^{-}}=\left(\Sigma x_{i}^{-}\right) / N^{-}, \\
& \Sigma x_{i}^{+}+\Sigma x_{i}^{-}=N \bar{x}=0,
\end{aligned}
$$

where the overbar denotes the average over the sample, $x_{i}^{+}$the positive values of $x$ and $x_{i}^{-}$the negative values.

The fractional area covered by the parcels with positive $x$ is:

$$
\varphi=N^{+} / N
$$

whereas the area covered by the parcels with negative $x$ is:

$$
1-\varphi=N^{-} / N
$$

The above relations imply:

$$
\overline{x^{+}} / \overline{x^{-}}=-N^{-} / N^{+}=(\varphi-1) / \varphi .
$$

If we assume that the aircraft groundspeed is almost constant during a run, and that the turbulent field has no preferred horizontal orientation, then the fractional time of a class of parcel does not significantly differ from the fractional area covered by this class. Figure 1 presents the fractional coverage of the negative parcels $(1-\varphi)$ for vertical velocity, potential temperature, specific humidity and virtual potential temperature (buoyancy). For randomly distributed turbulence, this term would be 0.5 . The altitude is normalised by the thickness of the mixed layer, $h$. As mentioned above, $h$ represents the thickness of the layer driven by the surface buoyancy flux. This layer does not reach the capping broken Sc layer, i.e. the two layers are decoupled (R95). In the lower half of the mixed layer, all the fractional coverages presented in Figure 1 exceed 0.5, which results from updrafts that occupy a smaller area but are stronger than downdrafts; similarly, warm parcels occupy a lower surface but are stronger (in temperature) than cold parcels; and moist parcels occupy a lower surface but are stronger (in moisture) than dry parcels; in other words, the skewness of vertical velocity, temperature and moisture is positive. The mathematical relation beween the fractional coverage and the skewness has been 

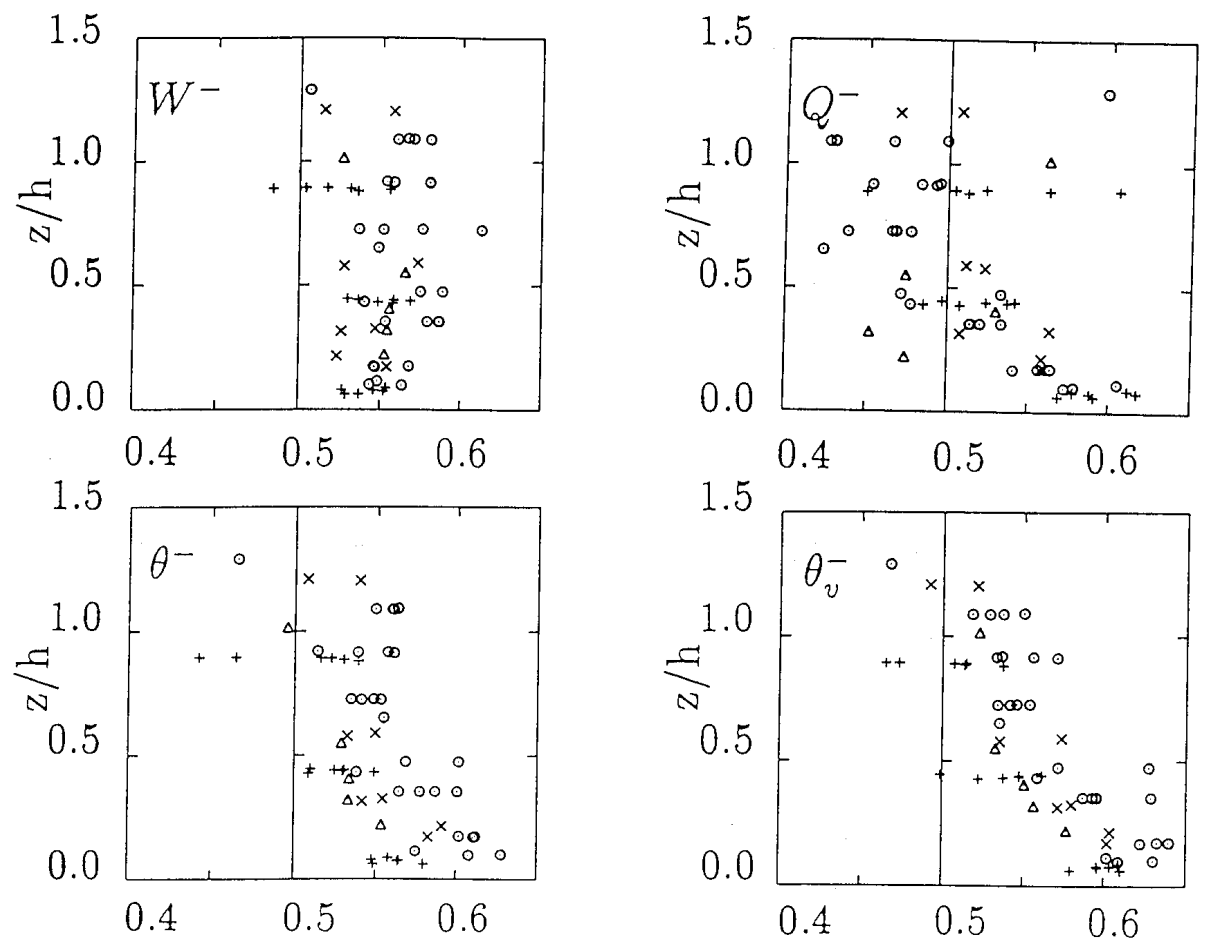

Figure 1. Fractional area covered by the parcels with a negative fluctuation in vertical velocity $\left(w^{-}\right)$, potential temperature $\left(\theta^{-}\right)$, specific humidity $\left(Q^{-}\right)$and buoyancy $\left(\theta_{v}^{-}\right)$, vs. the reduced altitude $z / h$. The various symbols correspond to the different missions: June $8(+)$, June $16(\triangle)$, June $19(\bigcirc)$ and June $20(\times)$. The general characteristics of these situations can be found in R95 (their Table I).

established in the case of a simple "bulk" model, which considers, for a variable $x$, only two parcels, the first having the value $x^{+}$and covering the fractional area $\varphi$, and the second having the value $x^{-}$, and covering the fractional area $1-\varphi$. Area averaging thus satisfies:

$$
x^{+} \varphi+x^{-}(1-\varphi)=\bar{x} .
$$

The skewness of $x, S_{x}$, is thus related to $\varphi$ by the following relation (see for instance Randall et al., 1992):

$$
\varphi=0.5-\frac{S_{x}}{2\left(4+S_{x}^{2}\right)^{1 / 2}} .
$$

It can be seen from (9) that an unskewed signal corresponds to $\varphi=0$, a positive skewness to $\varphi<0.5$ and a negative skewness to $\varphi>0.5$. For example, $S_{x}=0.5$ and $S_{x}=1.0$ correspond to $\varphi=0.379$ and $\varphi=0.276$ respectively; $S_{x}=-0.5$ and $S x=-1.0$ correspond to $\varphi=0.621$ and $\varphi=0.724$ respectively. The validity of (9) for the SOFIA data can be verified in comparing the results presented in 
Figure 1 with the skewness of $w, \theta$ and $Q$ presented in R95. The agreement is excellent.

The ratio $\overline{x^{+}} / \overline{x^{-}}$(see (7)) is also often used to characterize the relative importance of two kinds of parcels. For the vertical velocity, it can be deduced from the data presented in Figure 1 that this ratio is on average -1.25 for the whole mixed layer. Greenhut and Khalsa (1982) showed that this ratio varies according to the threshold chosen. For a threshold set to zero, as with the one we used, they found a value of -1.4 , which corresponds to their vertical velocity presenting a skewness greater than ours.

In the upper half of the mixed layer, the downdrafts continue to cover a greater area than the updrafts $(1-\varphi>0.5$ for $w)$, whereas for moisture $1-\varphi$ becomes very scattered and close to 0.5 on average. This behaviour is a consequence of the decoupling between the mixed layer and the Sc layer above: if the two layers were coupled, then the radiative cooling at the top of the cloud would result in negatively buoyant downdrafts, which would induce a negatively skewed vertical velocity in the lower layers (see for instance Randall et al., 1992). Furthermore, the strong negative discontinuity in moisture, at the top of the Sc layer, would induce (via entrainment processes) a negatively skewed moisture through most of the boundary layer (Deardorff, 1974; Frangi, 1979), which is not the case here. For potential temperature, the values remain greater than 0.5 , but significantly lower than in the lower half of the mixed layer. The result is that the buoyancy is positively skewed throughout the whole mixed layer, but with a continuous decrease from the surface to the top of the layer. Further interpretation requires multi-variate conditional sampling in order to identify the various parcels in terms of velocity and buoyancy.

\subsection{Multi-VARIATE CONDITIONAL SAMPLING}

In multi-variate conditional sampling, a parcel is defined according to several criteria. For example, an identical criterion can be applied simultaneously to several variables. Bi-variate conditional sampling can, for instance, be used to analyse the sensible heat transfer, by identifying the parcels according to the sign of their fluctuation in $w$ and $\theta$. Four quadrants can be so defined $\left(w^{+} \theta^{+}, w^{-} \theta^{-}, w^{-} \theta^{+}\right.$ and $w^{+} \theta^{-}$), the first two of them having a positive contribution to the flux and the other two negative. Such a bi-conditional sampling technique has been applied by R95 to the SOFIA data in order to analyse the sensible heat flux, latent heat flux and buoyancy flux. For each parcel, the fractional area as well as its contribution to the total flux has been analysed.

We propose here to go more deeply into the analysis of the turbulence structure by using a tri-variate conditional analysis. Knowing that the three main parameters controlling the vertical turbulent fluxes are the fluctuations in vertical velocity, temperature and moisture (the latter two, in SOFIA, contribute to the surface 
buoyancy with the same order of magnitude), the conditions relate to the sign of these parameters, and 8 classes were then defined: $w^{+} \theta^{+} Q^{+}, w^{+} \theta^{+} Q^{-}$, etc.

Figure 2 presents the fractional area covered by each of the 8 classes. In the lower part of the mixed layer, two classes cover the major area of the turbulent field: $w^{+} \theta^{+} Q^{+}$and $w^{-} \theta^{-} Q^{-}$, the latter being larger than the former. This corresponds to an organization of the turbulent field, which originates in the buoyancy at the surface: the air close to the sea surface being warmer and moister than above, this generates moist and warm updrafts. Conservation of mass implies that these updrafts be compensated by downdrafts, whose temperature and moisture are lower than the average temperature and moisture at a given altitude. The difference in the area covered by these two parcels can be explained as follows: an updraft accelerates when it rises from the surface to the upper levels because of its positive buoyancy (close to the surface, the potential temperature and moisture decrease as altitude increases). As opposed to this, downdrafts decelerate when approaching the surface (although their buoyancy remains negative) because of the constraint exerted by the surface (at the surface, $w=0$ ). In the end, it must be noted that the two kinds of parcels contribute to positive fluxes of sensible heat, latent heat and buoyancy. Each of the 6 remaining classes covers at most $10 \%$ of the total area. Among them, the two most significant are $w^{+} \theta^{-} Q^{-}$and $w^{-} \theta^{+} Q^{-}$. The former corresponds to parcels of "environment" which are entrained into buoyant updrafts, against their own buoyancy. The latter is composed of parcels originating from the upper part of the boundary layer, where the vertical gradient of potential temperature is positive and that of moisture is negative. The 4 remaining classes contain few parcels: detrainment of thermals $\left(w^{-} \theta^{+} Q^{+}\right)$is very scarce because the updrafts have a strong buoyancy; the origin of parcels with positive moisture and negative temperature $\left(w^{+} \theta^{-} Q^{+}\right.$and $\left.w^{-} \theta^{-} Q^{+}\right)$cannot be easily identified; the last class $\left(w^{+} \theta^{+} Q^{-}\right)$corresponds to parcels which came from the upper layers and have been entrained into updrafts.

When rising upwards through the mixed layer, this scenario is considerably modified. The area occupied by the positively buoyant thermals $\left(w^{+} \theta^{+} Q^{+}\right)$decreases, on the one hand because of the homogenization with the environment by turbulent mixing, and on the other hand because of the mean profiles of temperature and moisture. It has been shown, mainly by large eddy simulation of the boundary layer (see for instance Wyngaard and Brost, 1984), that the vertical gradients of potential temperature and moisture are not zero in the mixed layer, and are affected by the strong gradients which appear in the surface layer and at the top of the mixed layer. The resulting profile is continuously decreasing from the surface to the top of the mixed layer for specific humidity, and, for potential temperature, decreasing in the lower part and increasing in the upper part. The result is that a moist and warm thermal rising from the surface, when reaching the upper half of the mixed layer, remains moister but becomes colder than its environment. This corresponds to a transfer from the class $w^{+} \theta^{+} Q^{+}$towards $w^{+} \theta^{-} Q^{+}$when altitude increases. The other major class in the upper half of the mixed layer is $w^{-} \theta^{+} Q^{-}$. These parcels 

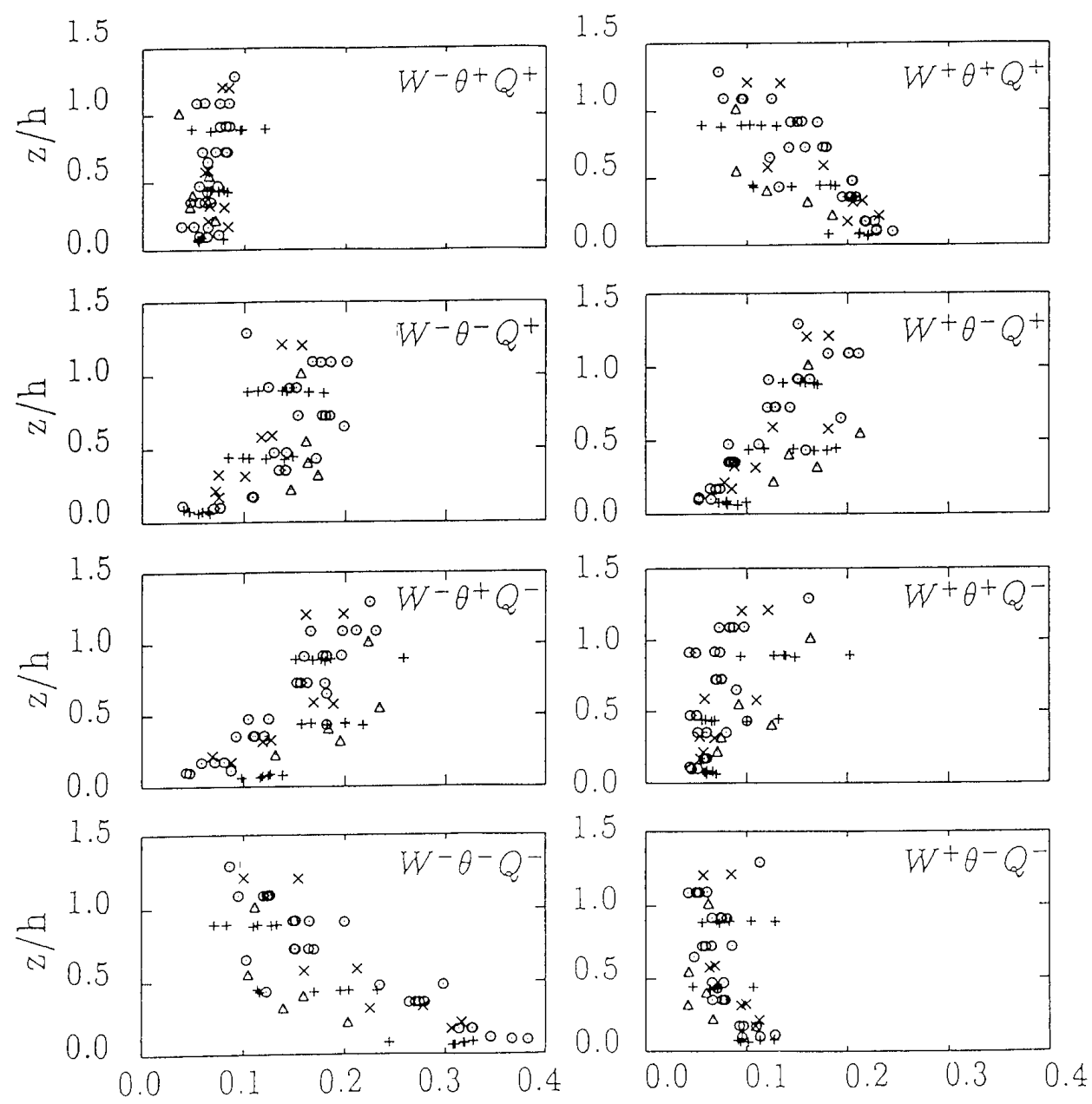

Figure 2. Fractional area covered by each of the eight parcels vs. the reduced altitude $z / h$. See R95 for symbols definition.

originate, partly, in the entrainment process produced by the moist updrafts at the top of the mixed layer, but also in entrainment at the top of the Sc capping layer generated by radiative cooling. However, the decoupling between the Sc layer and the mixed layer (as shown by R95) indicates to us that these latter parcels must be scarce.

Two other classes cover important areas in the upper half of the mixed layer: $w^{+} \theta^{+} Q^{-}$and $w^{-} \theta^{-} Q^{+}$. Considering the mean vertical gradients in temperature and moisture, it appears that these parcels have both been overturned: $w^{+} \theta^{+} Q^{-}$ originates from upper levels and $w^{-} \theta^{-} Q^{+}$from lower levels. For these two kinds of parcels, the buoyancy due to moisture acts in a way opposite to that of temperature. 
This could explain the ability of these parcels to be overturned by parcels with stronger buoyancy.

Throughout the whole mixed layer, the scarcest parcels are those whose thermal and moist buoyancy acts in the direction opposite to that of the vertical velocity $\left(w^{+} \theta^{-} Q^{-}\right.$and $\left.w^{-} \theta^{+} Q^{+}\right)$.

Figure 3 presents, for each of the 8 classes, the buoyancy flux transported by each class. The flux is normalized by the total surface buoyancy flux $\left(\overline{w^{\prime} \theta_{v}^{\prime}}\right)_{0}$. Another

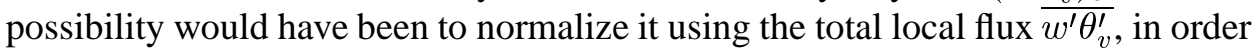
to exhibit the fractional contribution of each class to the local flux. This technique has been used by R95 with bi-variate conditional sampling. The problem is that, above $0.5 \mathrm{~h}$, the buoyancy flux becomes very low (see R95), and the results become all the more scattered and difficult to interpret when the number of conditions (i.e. the number of classes) increases. In the lower half of the mixed layer, it can be seen on Figure 3 that the major part of the flux results from the two classes $w^{+} \theta^{+} Q^{+}$ and $w^{-} \theta^{-} Q^{-}$. These two classes are those which also cover the greatest area (see Figure 2). However, the greatest area is occupied by $w^{-} \theta^{-} Q^{-}$whereas the greatest contribution to the buoyancy flux is brought by $w^{+} \theta^{+} Q^{+}$. This can be related to the buoyancy source of turbulence, which lies in the temperature and moisture gradients close to the surface. These gradients generate the moist and warm updrafts which carry therefore most of the buoyancy.

The 6 remaining classes present low values. In the lower part of the mixed layer, detrainment of thermals $\left(w^{-} \theta^{+} Q^{+}\right)$and entrainment of the environment into thermals $\left(w^{+} \theta^{-} Q^{-}\right)$bring the major contribution to the negative buoyancy flux. The last four classes present an opposite sign for temperature and moisture. It can be inferred from the sign of the flux that the buoyancy is generally of the same sign as that of the temperature (for example, $w^{-} \theta^{-} Q^{+}$presents a positive buoyancy flux, which implies a negative buoyancy), except for $w^{-} \theta^{+} Q^{-}$in the lower layers.

\section{Scale Analysis}

Spectral analysis (in particular analysis of the characteristic length scales) is a tool which is widely used to bring more insight into the internal structure of the turbulent field. Filtering techniques are frequently applied to turbulence signals, in order to remove large scale trends (high-pass filtering), to avoid aliasing, to remove high-frequency noise (low-pass filtering), or to exhibit the characteristics of specified scales (band-pass filtering). Combining filtering techniques with conditional sampling analysis could therefore allow a precise description of the boundary-layer turbulence. For example, Mahrt and Paumier (1984), in their analysis of AMTEX data (boundary layer over the sea with strong surface fluxes of heat and momentum), have decomposed their signals in three bands according to the following cut-off wavelengths $\lambda: \lambda<200 \mathrm{~m}, \lambda>2000 \mathrm{~m}$, and $200 \mathrm{~m}<\lambda<2000 \mathrm{~m}$. Using 

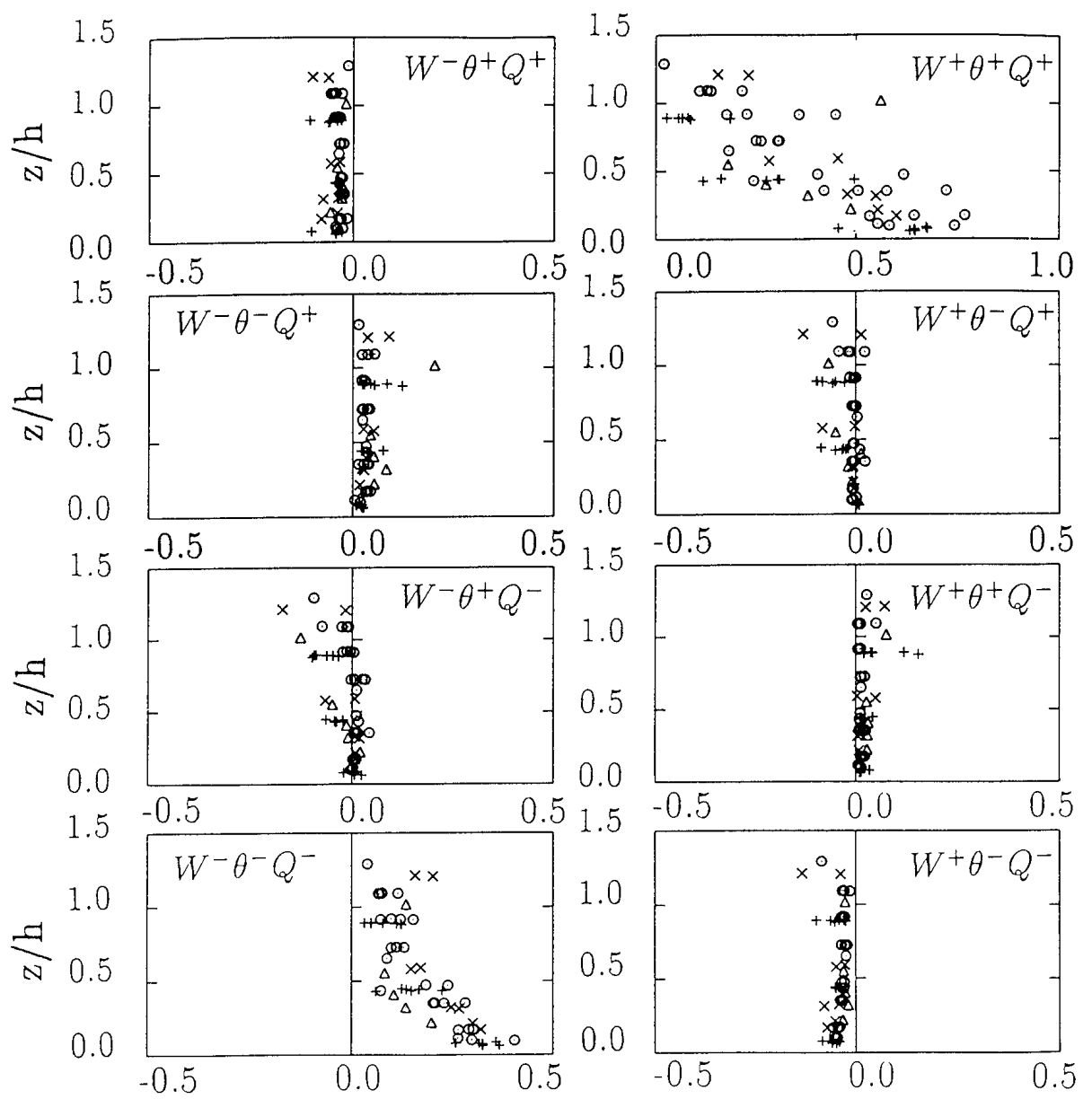

Figure 3. Contribution to the buoyancy flux of each of the eight parcels vs. the reduced altitude $z / h$. The data are normalized by the surface buoyancy flux. Note that the abscissa scale on the right upper graph is different from those of the other graphs.

the filtered signals, they applied a bi-variate conditional sampling technique, and analysed the transfer of buoyancy in this way. A similar technique has been applied by Chou and Zimmerman (1989) for the GALE data, corresponding to a cold air outbreak (strong sensible and latent heat fluxes, and moderate winds). The cut-off wavelengths were the same as those of Mahrt and Paumier (1984). In these two studies, these wavelengths do not therefore vary according to altitude.

We have performed an analysis of the SOFIA data based on the same principle. The first problem was to define the number of wavelengths bands, as well as the values for the cut-off wavelengths. These were chosen after a spectral analysis of the signals: it is now well known that spectra and cospectra vary in the boundary layer according to altitude. For example, the wavelength of the vertical velocity 
spectrum peak $\lambda_{m}$, which is a characteristic scale of turbulence, scales with altitude. For the convective boundary layer, this scale increases from zero at the surface up to a maximum value in the middle of the boundary layer. This maximum is slightly greater than the boundary-layer thickness (Frangi, 1979; Caughey and Palmer, 1979). Above, $\lambda_{m}$ diminishes because of the barrier effect of the capping inversion, or remains quite constant (Kaimal et al., 1976). Lenschow and Stankov (1986) found no significant decrease in the vertical velocity integral scale near the top of the convective boundary layer. This scale is closely related to the wavelength of the vertical velocity spectrum peak. In the case of SOFIA, we have shown (R95) that the top of the mixed layer is not marked by a sharp inversion. Accordingly, a detailed exploration of the spectral characteristics of the signals (Réchou, 1995) has shown that $\lambda_{m}$ increases from the surface up to the top of the mixed layer. The various cut-off wavelengths were thus chosen proportional to the altitude and not to the MABL thickness (this choice would probably have been different for a continental convective boundary layer topped by a sharp inversion. In this case, the spectral length scales vary with the boundary-layer thickness rather than with altitude). The reduced cut-off wavelengths $\lambda_{i}=\mathrm{TAS} /\left(n_{i} z\right)$ (where $n_{i}$ is the frequency in the aircraft time reference, $z$ the altitude of the sample and TAS the mean true airspeed of the aircraft along the sample) are therefore constant with altitude. Four cut-off reduced wavelengths $\left(\lambda_{1}, \lambda_{2}, \lambda_{3}\right.$ and $\left.\lambda_{4}\right)$ are defined for each sample, delimiting five bands ( $B_{1}$ to $B_{5}$ ), according to increasing wavelengths). From the spectral analysis of the signals performed by Réchou (1995), it appears that $B_{3}$ corresponds to the maximum of the cospectra of sensible heat, latent heat and momentum. On a logarithmic scale, the bands $B_{2}, B_{3}$ and $B_{4}$ have the same width: $\lambda_{i+1} / \lambda_{i}=10^{0.6} \approx 4$, where $\lambda_{i}$ and $\lambda_{i+1}$ are the extreme wavelengths of the band $B_{i+1}$. The chosen values for the cut-off reduced wavelengths are: $\lambda_{1}=10^{-0.4}, \lambda_{2}=10^{+0.2}, \lambda_{3}=10^{+0.8}$ and $\lambda_{4}=10^{+1.4}$, and the corresponding wavelength bands, $B_{1}$ to $B_{5}$ are:

$$
\left.\begin{array}{ll}
B_{1}: & \lambda_{r}<\lambda_{1} \\
B_{2}: & \lambda_{1} \leq \lambda_{r}<\lambda_{2} \\
B_{3}: & \lambda_{2} \leq \lambda_{r}<\lambda_{3} \\
B_{4}: & \lambda_{3} \leq \lambda_{r}<\lambda_{4} \\
B_{5}: & \lambda_{r} \geq \lambda_{4}
\end{array}\right\},
$$

where $\lambda_{r}$ is the reduced wavelength. As an example, for an altitude $z=100 \mathrm{~m}$, the non-reduced corresponding wavelengths $(\lambda=$ TAS $/ n)$ are $40 \mathrm{~m}, 160 \mathrm{~m}, 640 \mathrm{~m}$ and $2560 \mathrm{~m}$. $B_{1}$ thus lies within the inertial subrange, whereas $B_{5}$ corresponds to low-frequency movements which are generally removed for turbulence moments computation. For higher altitude runs, $\lambda_{4}$ is sometimes of the same order as (or greater than) the length of the run and the low-pass filtering in $B_{5}$, and band-pass filtering in $B_{4}$, cannot therefore be applied. In practice, this limit was fixed to $10000 \mathrm{~m}$. This explains why some points are missing on the B5 and B4 plots in the following figures. 


\subsection{FLUXES}

Figure 4 presents the profiles of the sensible and latent heat flux within the various bands. The total flux is also presented. The so-called "total" flux in fact corresponds to the flux computed by the eddy-correlation technique after high-pass filtering with a cut-off frequency of $0.024 \mathrm{~s}^{-1}$, as explained in R95. (This filtering has been applied to the signals before computing each turbulence moment; the "total" moments presented in the following figures correspond therefore to data so filtered). The data of Figure 4 are normalized by the total surface flux, which can be expressed in terms of convective scales, defined as (Deardorff, 1970):

$$
\begin{aligned}
& w_{*}=\left[\left(g / T_{0}\right) \overline{w^{\prime} \theta_{v_{0}}^{\prime}} h\right]^{1 / 3} \\
& \theta_{*}=\overline{w^{\prime} \theta_{0}^{\prime}} / w_{*} \\
& Q_{* *}=\overline{w^{\prime} Q_{0}^{\prime}} / w_{*},
\end{aligned}
$$

where $g$ is gravity and $T_{0}$ the surface temperature. Most of the flux occurs in the bands $B_{3}$ and $B_{4}$, for which the profiles appear quite similar to (although weaker than) the profile of the total flux. No flux appears in $B_{1}$, which confirms the fact that the inertial subrange does not significantly contribute to the transfers (from dimensional analysis, it can be shown that the sensible heat and latent heat flux cospectra follow a $-7 / 3$ power law in the inertial subrange, and therefore that the corresponding flux quickly decreases). In $B_{2}$, the transfers, although weak, appear to be much less scattered than in the neighbouring band $B_{3}$. The profile of the latent heat flux is remarkably constant with altitude. In fact, this band contains small scale updrafts, whose principal origin lies in the surface layer. They grow when rising through the boundary layer, and continue to transfer the same amount of water vapor.

If the transfers are well "bounded" on the high-frequency side (they vanish in $B_{1}$ ), they are far from being weak on the low-frequency side. Keeping in mind that $B_{3}$ is approximately centered around the cospectral peak, it appears from $B_{4}$ and $B_{5}$ that the cospectral behaviour is far from symmetrical, and that the low frequencies significantly contribute to the transfers. In particular, $B_{5}$, which corresponds to wavelengths greater than about 25 times the altitude $z$, presents considerable but very scattered values. Such low-frequency eddies, which cannot be related to three-dimensional isotropic turbulence, have therefore to be taken into account when studying the transfers in the boundary layer. However, if they are included in statistical computations, as in the eddy-correlation method, they induce considerable scatter in the results. That's why they are generally removed by high-pass filtering of the signals. This problem has been thoroughly discussed in Lenschow and Stankov (1986), Lenschow et al. (1994) and Mann and Lenschow (1994). They have shown, from theoretical considerations as well as from airborne 

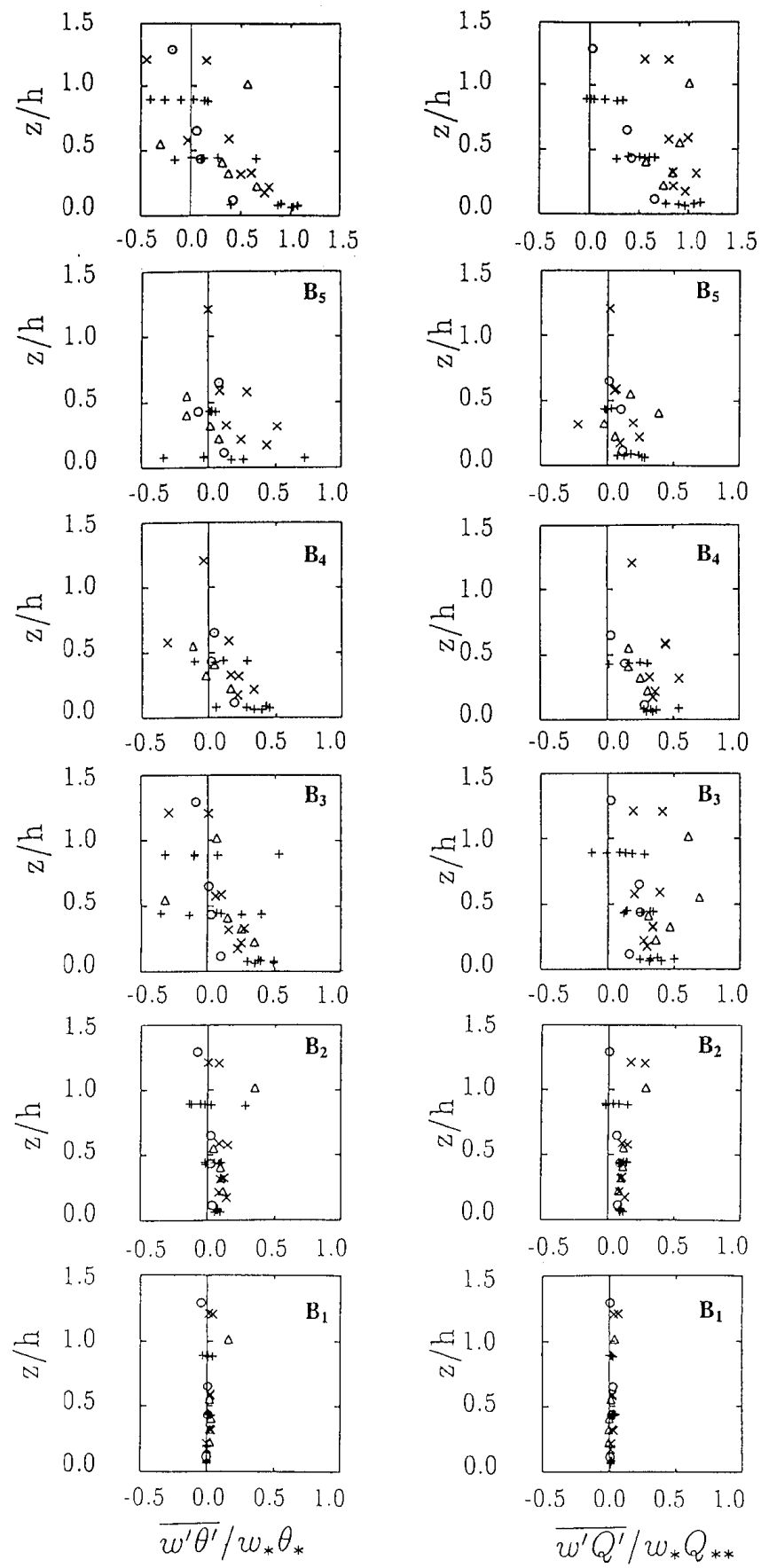

Figure 4. Contribution of the various bands (from bottom to top: $B_{1}$ to $B_{5}$ ) to the sensible heat flux (left) and the latent heat flux (right) vs. the reduced altitude $z / h$. The uppermost graph represents the profile of the entire flux. The data are normalized by the surface flux. Note that the abscissa scale on the uppermost graphs is different from those of the other graphs. 
measurements in the boundary layer, that one has to make a "compromise", when computing turbulent fluxes by the eddy-correlation technique, between a "random" error which reduces when the cut-off frequency of the high-pass filtering increases, and a "systematic" error which grows with cut-off frequency.

\subsection{VARIANCES}

The variances of the horizontal and vertical wind components are presented in Figure 5 . They are normalized by the convective velocity scale $w_{*}$, because it was demonstrated by R95 that this parameterization is well adapted to the SOFIA data. From $B_{1}$ to $B_{3}$, the behaviour of the horizontal and vertical components appear quite similar, with the energy increasing according to the wavelengths. Furthermore, for a particular bandwidth, the energy increases according to the altitude, from the surface up to the middle of the boundary layer. The small eddies (of wavelength smaller than that of the vertical velocity spectrum peak) therefore appear characteristic of a three-dimensional isotropic turbulence whose energy and size of eddies increase according to the distance to the surface. Furthermore, the energy in $B_{1}$ increases with altitude according to a $z^{2 / 3}$ law for the lower layers. This can be explained as follows: in the inertial subrange, the energy spectrum $S(n)$ is proportional to $n^{-5 / 3}$, according to the well-known Kolmogorov formulation, which implies that the total energy in the band $B_{1}$ can be expressed as:

$$
\sigma_{B_{1}}^{2}=\int_{n_{1}}^{n_{q}} S(n) \mathrm{d} n=a \epsilon^{2 / 3}\left(n_{1}^{-2 / 3}-n_{q}^{-2 / 3}\right) \approx a \epsilon^{2 / 3}\left(n_{1}^{-2 / 3}\right),
$$

where $n_{q}$ is the Nyquist' frequency (for example, $8 \mathrm{~s}^{-1}$ for the Fokker 27), $a$ is a constant including among others the TAS of the aircraft and the Kolmogorov' constant, and $\epsilon$ is the TKE dissipation rate. Knowing that $n_{1}=\mathrm{TAS} /\left(\lambda_{1} z\right)$, (14) reduces to:

$$
\sigma_{B_{1}}^{2} \approx b \epsilon^{2 / 3} z^{2 / 3}
$$

where $b$ is a constant. For the altitudes of between $50 \mathrm{~m}$ (the lowest flight level) and the middle of the boundary layer, it has been shown by R95 that $\varepsilon$ does not vary significantly (in other words, in the inertial subrange, for a given frequency, the spectrum energy does not vary with altitude). It can therefore be seen from (15) that the energy in $B_{1}$ follows a $2 / 3$ power law.

The isotropy between horizontal and vertical components remains up to $B_{3}$ band, i.e. down to the frequencies corresponding to the maximum of the vertical velocity spectrum. For lower frequencies, the energy of the vertical component decreases, whereas that of the horizontal remains high even for the lowest altitudes. When considering the total variance of each component, it increases according to the altitude following the classical 2/3 power law (Kaimal and Finnigan, 1994) for the vertical, whereas it remains quite constant or even decreases for the horizontal. 

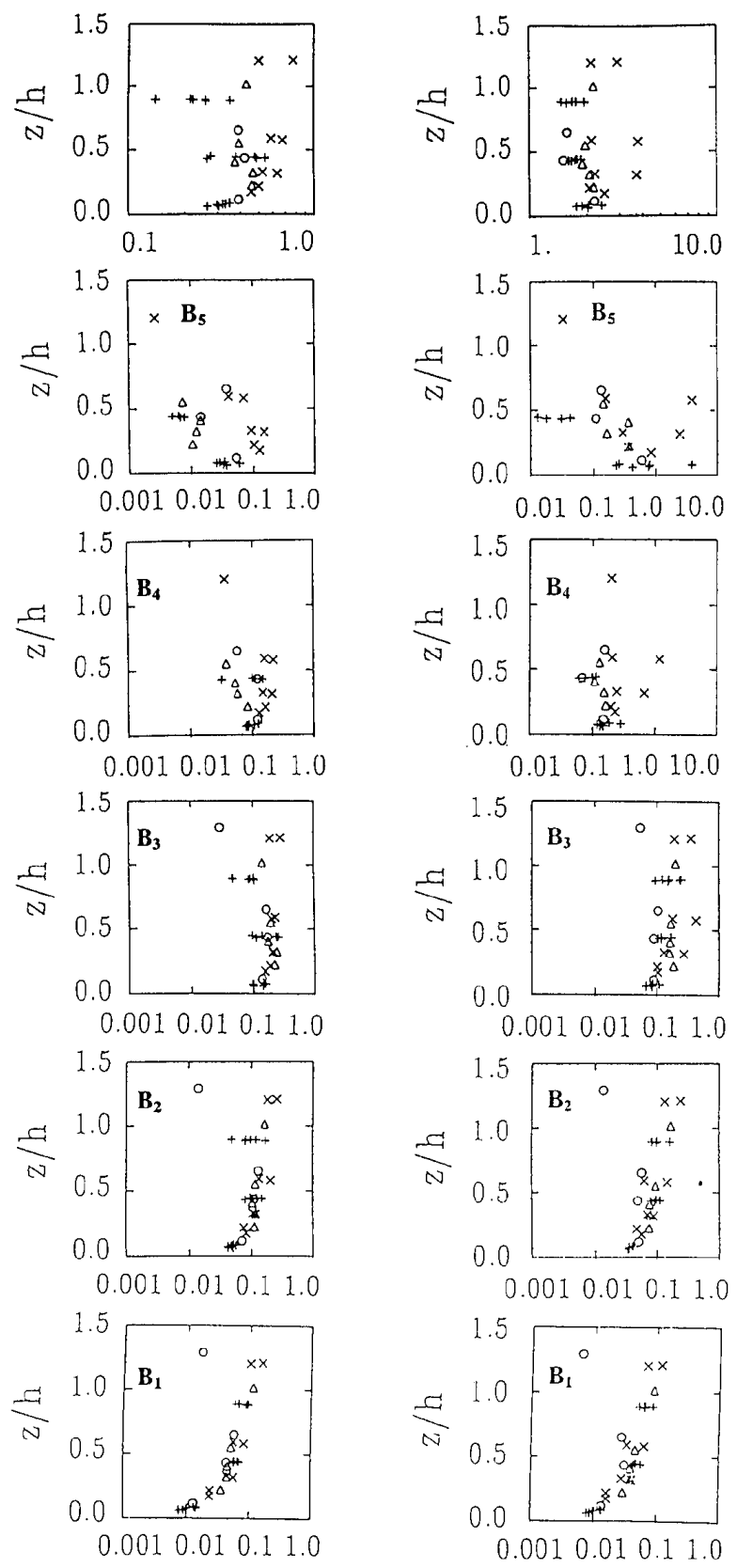

$$
\overline{w^{2}} / w_{*}^{2}
$$$$
\left(\overline{u^{\prime 2}}+\overline{v^{\prime 2}}\right) /\left(2 w_{*}^{2}\right)
$$

Figure 5. Identical to Figure 4, except for the vertical (left) and horizontal (right) velocity variance. Note that the abscissa scale on the uppermost graphs is different from those of the other graphs. 
This is explained by the fact that the vertical eddies scale with altitude, whereas the horizontal eddies scale with the boundary-layer thickness (Garratt, 1992; Kaimal and Finnigan, 1994).

Figure 6 presents the potential temperature and moisture variance profiles. They are normalized by the convective scales. In the inertial subrange, we can deduce, by analogy with the velocity components, that the energy in the inertial subrange can be expressed as:

$$
\begin{aligned}
& \sigma_{B_{1}}^{2} \approx a_{T}^{1} \epsilon^{-1 / 3} N_{T} z^{2 / 3}=a_{T}^{2} C_{T}^{2} z^{2 / 3} \quad \text { for temperature, and } \\
& \sigma_{B_{1}}^{2} \approx a_{Q}^{1} \epsilon^{-1 / 3} N_{Q} z^{2 / 3}=a_{Q}^{2} C_{Q}^{2} z^{2 / 3} \quad \text { for moisture }
\end{aligned}
$$

where $N_{T}$ and $N_{Q}$ are the dissipation rates of half-variance of potential temperature and moisture respectively, $C_{T}^{2}$ and $C_{Q}^{2}$ the structure parameters for temperature and moisture respectively, and $a_{T}^{1}, a_{T}^{2}, a_{Q}^{1}$ and $a_{Q}^{2}$ are constants. For continental boundary layers under free convection conditions with weak entrainment, it has been shown (see for instance Weill et al., 1980; Wyngaard, 1983) that $C_{T}^{2}$ follows a $z^{-4 / 3}$ law up to the upper part of the boundary layer. When the entrainment becomes important this law is no longer valid above $0.5 \mathrm{~h}$ (Druilhet et al., 1983), or even above $0.2 \mathrm{~h}$ (Wyngaard, 1983). Data concerning $C_{Q}^{2}$ are much more scarce. Lenschow et al. (1980) found that, over the sea with strong surface heat and momentum fluxes, this law is only valid up to $0.1 \mathrm{~h}$. According to (16) and (17), a $-4 / 3$ power law of $C_{T}^{2}$ and $C_{Q}^{2}$ would give a $-2 / 3$ power law for the total energy in the band $\mathrm{B}_{1}$. As can be seen in the figure, this is not the case. This is consistent with the behaviour of $C_{Q}^{2}$ which, as shown by Réchou (1995), does not follow the $z^{-4 / 3}$ law. In the SOFIA case, entrainment cannot be invoked to explain this departure. Due to the low signal away from the surface, the temperature spectra are somewhat noisy in the high-frequency region and could cause an overestimation of $C_{T}^{2}$. But this problem does not arise on the moisture sensor (Lyman- $\alpha$ device), and the behaviour of the temperature and moisture variance profiles are very similar (although a little more scattered for the former than for the latter).

Although not yet satisfactorily explained, the profiles in $B_{1}$ are well defined. When moving towards lower frequencies, the scatter of the results increases, together with the energy involved in the band. This is because the energy spectra are not bounded on the low-frequency side (given the wavelengths sampled, i.e. the run length), although the surface conditions were horizontally quite homogeneous (sea surface temperature in particular). The considerable scatter in $B_{5}$ (two orders of magnitude) results from the variability in the low-frequency spectra behaviour and the importance of "random" error in the calculation of variance, and therefore demonstrates the value of high-pass filtering. 

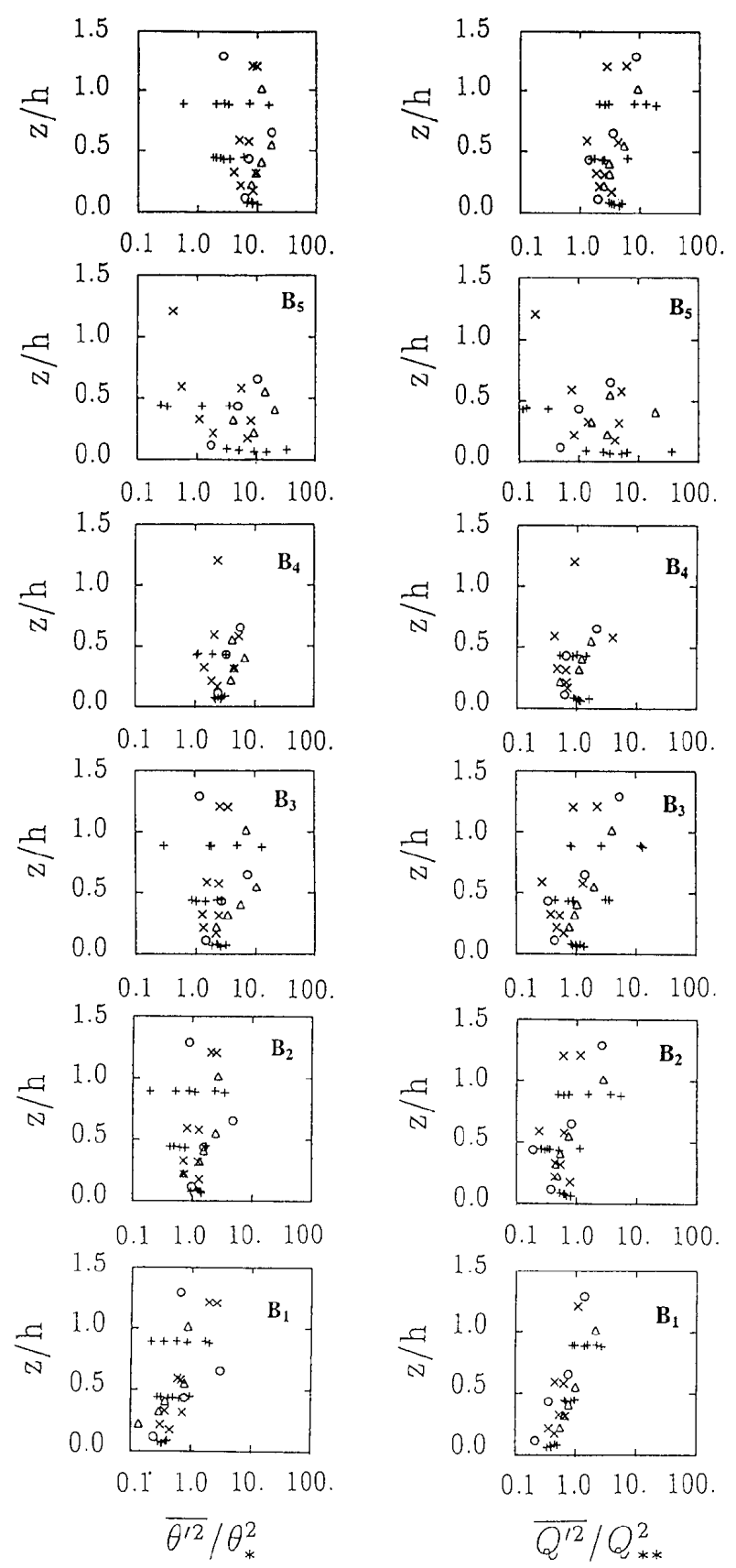

Figure 6. Identical to Figure 4, except for the potential temperature (left) and specific humidity (right) variance. 


\section{Conditional Sampling on Filtered Signals}

The conditional sampling technique, as described in a previous section, has been applied to the signals filtered in the 5 bands described above. The tri-variate conditional technique has been applied to the vertical velocity $w$, the potential temperature $\theta$ and the moisture $Q$. Eight classes were therefore defined for each run, according to whether $w, \theta$ and $Q$ were positive or negative. A flux $\overline{w^{\prime} x^{\prime}}$ can therefore be expressed as (if we assume ideal cut-off filters):

$$
\overline{w^{\prime} x^{\prime}}=\frac{1}{N} \sum_{j} \sum_{k} \sum_{i} w_{i j k}^{\prime} x_{i j k}^{\prime},
$$

where $i$ is the current index along the sample, $j$ relates to the band $B_{j}$ and $k$ to the class according to the sign of $w, \theta$ and $Q$.

Figure 7 presents the profiles of the fractional area covered by each class for the various bands $B_{1}$ to $B_{5}$. In order to keep the legibility of the figure, the individual runs are replaced by the averages in overlapping layers of $0.2 \mathrm{~h}$ thickness. The eight classes can thus be represented on the same graph. For randomly distributed turbulence, without internal organization or transfers, each class would cover the same area, which is almost the case in the inertial subrange $\left(B_{1}\right)$. It can be noted, however, that the two classes $w^{+} \theta^{+} Q^{+}$and $w^{-} \theta^{-} Q^{-}$(which cover the major area: see Figure 2 ) are, even in $B_{1}$, slightly greater than the others, which indicates the beginning of the organization of the turbulence. This organization increases through $B_{2}$ and $B_{3}$, and becomes important in $B_{4}$. The profiles in $B_{2}, B_{3}$ and $B_{4}$ are close to those computed from the "entire" signals, also presented on the figure. The conclusions which can be drawn from these bands are therefore quite similar to those presented above, in the comments on Figure 2. In the very low frequency band $\left(B_{5}\right)$, a certain organization remains: the parcels $w^{-} \theta^{+} Q^{+}$and $w^{+} \theta^{-} Q^{-}$are very scarce throughout the whole mixed layer, whereas they appear to be much more numerous close to the surface at smaller scales. Such parcels, having a buoyancy opposite to their velocity, have been entrained by, or detrained from stronger currents, which is only possible for small parcels. It is also interesting to note that, in $B_{5}$, the six remaining classes cover an almost similar area in the lower layers, whereas they are clearly separated in the upper part. In fact, these parcels of great scale (much greater than the TKE production scales) are mixed in the lower layers because of small scale energetic turbulence and because of the reduction of characteristic scales imposed by the proximity of the surface. On the contrary, in the upper part of the mixed layer, they can exist. The dominant parcels are $w^{+} \theta^{-} Q^{+}$and $w^{-} \theta^{+} Q^{-}$i.e., given the vertical gradients of temperature and moisture in this region, the sign of the velocity is coherent with those of temperature and moisture. In other words, an updraft originates from the lower layers, whereas a downdraft originates from the upper layers.

Another point can be made from the profiles in the various bands. It appears that the profiles of two classes of opposite sign $\left(w^{s 1} \theta^{s 2} Q^{s 3}\right.$ and $w^{-s 1} \theta^{-s 2} Q^{-s 3}$, where 


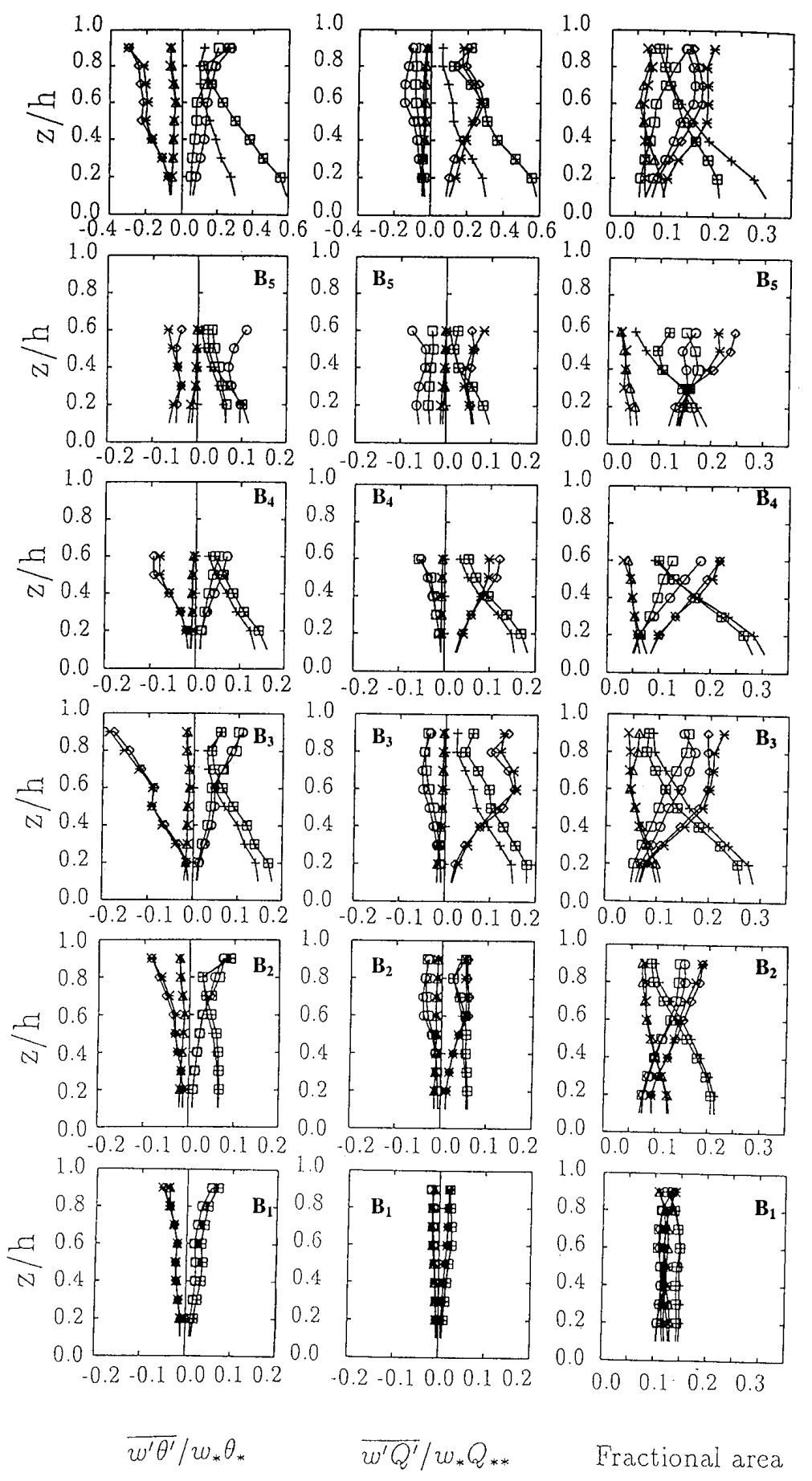

Figure 7. Right: fractional area covered by each of the eight parcels in the various bands (from bottom to top: $B_{1}$ to $B_{5}$ ) vs. the reduced altitude $z / h$. The uppermost graph represents the fractional area covered by each parcel computed on the "entire" signal (i.e., high-pass filtered with the systematic cut-off of $0.024 \mathrm{~s}^{-1}$ ). Middle: identical but for the latent heat flux (data normalized by the surface flux). Left: identical but for the sensible heat flux. The various symbols correspond to the following parcels: $w^{-} \theta^{-} Q^{+}: \bigcirc ; w^{+} \theta^{+} Q^{+}: \boxplus ; w^{-} \theta^{+} Q^{-}: * ; w^{+} \theta^{-} Q^{-}: \triangle ; w^{+} \theta^{-} Q^{+}: \diamond ; w^{-} \theta^{+} Q^{+}: \times$; $w^{+} \theta^{+} Q^{-}: \square ; w^{-} \theta^{-} Q^{-}:+$. Note that the abscissa scale on the uppermost graphs is different from those of the other graphs. 
$s_{i}(i=1,2$, or 3$)$ is the sign and $-s_{i}$ the opposite sign $)$ are close, whereas this is not true for the entire signal. For example, the difference between $w^{+} \theta^{+} Q^{+}$and $w^{-} \theta^{-} Q^{-}$is greater on the entire signal than in the various bands. This is due to the filtering, which tends to symmetrize the signals: the shape of the eddies cannot be reproduced accurately by a limited number of harmonics. In particular, skewness is reduced. In this sense, composite analysis based on a wavelet transform with the appropriate analysing function could provide more information, but would also be more complex to develop and interpret.

The contribution of the eight classes in the various bands to the fluxes of sensible heat and latent heat are also presented in Figure 7 . The fluxes are normalized by the total surface flux. As mentioned above, two parcels of opposite sign are more "symmetrical" in the various bands than in the entire domain. The scheme of the entire domain is well reproduced in the bands $B_{3}$ and $B_{4}$, which are very similar. In $B_{1}$, the various contributions are very weak, whereas in $B_{5}$ they are more important. In $B_{3}$, which corresponds to the scales of TKE production, like in $B_{4}$, two parcels become important in the upper part of the mixed layer: $w^{+} \theta^{-} Q^{+}$and $w^{-} \theta^{+} Q^{-}$. These parcels contribute to strong negative sensible heat flux, and positive latent heat flux.

The contribution of the various parcels to the fluxes at $z=0.25 \mathrm{~h}$ are indicated in Table I. In order to reduce the complexity of the picture, the parcels have been regrouped two by two: for example, $w^{s 1} \theta^{s 2}$ represents the sum of the contributions of $w^{s 1} \theta^{s 2} Q^{+}$and $w^{s 1} \theta^{s 2} Q^{-}$, whereas $w^{s 1} Q^{s 2}$ represents the sum of $w^{s 1} \theta^{+} Q^{s 2}$ and $w^{s 1} \theta^{-} Q^{s 2}$ (in other words, these results correspond to a bi-variate conditional sampling). The fluxes are normalized by the total surface flux. The flux transferred within a band is therefore the sum of the four quadrants corresponding to this band. We have also indicated the flux transferred by each quadrant, without filtering the signals in the various bands. It can be noted that the tranfers are greatest in $B_{3}$ and $B_{4}$ for both fluxes, and remain important in $B_{5}$ principally for the sensible heat flux. The symmetrization effect of the filtering, mentioned above, can here be seen through the difference between $w^{+} x^{+}$and $w^{-} x^{-}$which is greater for the whole signal than for the sum of the various bands. This table also exhibits the importance of the low frequencies on the transfers. The flux computed from the sum in the various bands and in the various classes (right-hand side term in (18)) is greater than the flux computed by the simple eddy-correlation technique with the high-pass filtering systematically used; for sensible heat flux, the latter is 0.54 , whereas it reaches 0.71 for the former. The difference between the two $(0.17)$ is approximately the same amount as the flux transferred in band $B_{5}$. A similar result can be found for latent heat flux, for which, however, this difference is only 0.09.

These low-frequency transfers result from meso scale circulations, whose role can be shown by the relation between the mixed-layer structure and the thermodynamic mean fields. For example, it has been shown by Weill (private communication), that the mixed-layer thickness is more related to to the mean temperature and moisture than to the surface heat fluxes. These thermodynamical profiles are 
Table I

Quantities transferred at $z=0.25 \mathrm{~h}$ by each parcel, in the various bands, for the sensible heat flux (upper part) and latent heat flux (lower part). The total of the five bands is also indicated. The last line of each part corresponds to the flux computed from signals high-pass filtered with the cut-off of $0.024 \mathrm{~s}^{-1}$, which is systematically used before eddy-correlation computation. All the data are normalized by the surface flux (extrapolated towards the surface from the profile of the flux computed from the signals high-pass filtered at a cut-off of $0.024 \mathrm{~s}^{-1}$ ).

\begin{tabular}{llllll}
\hline & $w^{+} \theta^{+}$ & $w^{+} \theta^{-}$ & $w^{-} \theta^{+}$ & $w^{-} \theta^{-}$ & Total \\
\hline$B_{1}$ & 0.03 & -0.03 & -0.03 & 0.03 & 0.01 \\
$B_{2}$ & 0.08 & -0.03 & -0.03 & 0.08 & 0.10 \\
$B_{3}$ & 0.17 & -0.04 & -0.04 & 0.15 & 0.24 \\
$B_{4}$ & 0.14 & -0.03 & -0.03 & 0.12 & 0.20 \\
$B_{5}$ & 0.14 & -0.05 & -0.05 & 0.13 & 0.17 \\
$B_{1}+B_{2}+B_{3}+B_{4}+B_{5}$ & 0.56 & -0.18 & -0.18 & 0.51 & 0.71 \\
$n>0.024 s^{-1}$ & 0.53 & -0.16 & -0.17 & 0.34 & 0.54 \\
\hline & $w^{+} Q^{+}$ & $w^{+} Q^{-}$ & $w^{-} Q^{+}$ & $w^{-} Q^{-}$ & Total \\
\hline$B_{1}$ & 0.02 & -0.01 & -0.01 & 0.02 & 0.02 \\
$B_{2}$ & 0.07 & -0.02 & -0.02 & 0.07 & 0.10 \\
$B_{3}$ & 0.20 & -0.03 & -0.03 & 0.17 & 0.31 \\
$B_{4}$ & 0.20 & -0.02 & -0.02 & 0.18 & 0.34 \\
$B_{5}$ & 0.12 & -0.07 & -0.04 & 0.09 & 0.10 \\
$B_{1}+B_{2}+B_{3}+B_{4}+B_{5}$ & 0.61 & -0.15 & -0.12 & 0.53 & 0.87 \\
$n>0.024 \mathrm{~s}^{-1}$ & 0.58 & -0.10 & -0.08 & 0.38 & 0.78 \\
\hline
\end{tabular}

affected by mean vertical motion (subsidence in anticyclonic areas, as in the region of SOFIA/ASTEX).

\section{Conclusion}

Turbulence airborne measurements performed during the SOFIA experiment have been used to analyse the lower part of the MABL (i.e., the mixed layer which is driven by surface fluxes and is, in the SOFIA case, decoupled from the Sc layer above). This study extends the work of R95, which presented the parameterization of the main turbulence characteristics. The results presented here were obtained by a conditional sampling technique, used to identify the various parcels of the turbulent field in the mixed layer, and to quantify the fractional area they cover as well as their contribution to the transfers of sensible heat, latent heat and buoyancy.

As a first approach, a single-variate conditional technique has shown the organization of the turbulence field into moist and warm updrafts which cover an area smaller than the downdrafts in the lower half of the mixed layer. This scheme is standard for a mixed layer driven by surface buoyancy. In the upper part, however, 
the results are representative of a mixed layer decoupled from the above Sc layer (the vertical velocity remains positively skewed, and the moisture skewness vanishes), and therefore significantly differs from a convective boundary layer with strong entrainment at its top (which results in negatively skewed moisture through most of the mixed layer); they also differ from a coupled, Sc topped, layer which presents negatively skewed vertical velocity in its upper half, resulting from the negative buoyancy at the top of the Sc layer due to radiative cooling.

From multi-variate conditional sampling, based on simultaneous criteria for vertical velocity, temperature and moisture, it has been shown that moist and warm updrafts generated close to the surface bring the major contribution to buoyancy flux in the lower half of the mixed layer. Dry and cool downdrafts that cover the greatest area make a considerable contribution to flux, but less than the moist and warm updrafts. For parcels having fluctuations in temperature and moisture of the opposite sign, it appears that buoyancy is generally of the same sign as the temperature fluctuation, although surface buoyancy flux is almost equally due to sensible heat and latent heat flux (R95).

The turbulence signals along the straight and level runs have been filtered into five bands defined according to the characteristic length scales of the turbulence. The central band corresponds to the maximum of the vertical velocity energy spectrum, and also to the maximum of sensible heat flux and latent heat flux cospectra. If the energy and the transfers are weak in the high-frequency range (inertial subrange), they remain important in the low-frequency range, except for vertical velocity variance. In particular, sensible heat flux and latent heat flux are quite large although very scattered. High-pass filtering of the turbulence signals, currently used for removing large scale trends before computing turbulent fluxes, therefore results in scatter reduction but also in the removal of a significant fraction of the flux.

Finally, the conditional sampling technique has been applied to the signals filtered in the various bands. It appears that the general organization of the turbulent field is well reproduced at the scales corresponding to the turbulence production ( $B_{3}$ band), despite a slight "symmetrization" of the signals due to the filtering operation. At the greatest scales $\left(B_{5}\right)$, an organization remains, mainly in the upper part of the mixed layer, although different from that of the production scale. At an altitude of $0.25 \mathrm{~h}$, the contribution of this band to sensible and latent heat flux is as high as (or greater than) 0.1 times the corresponding surface flux.

\section{Acknowledgements}

We want to express our gratitude to Alain Weill, who manages the SOFIA program, and to all the colleagues who contributed to the experiment. We are very grateful to people from the "Institut Géographique National", the "Division Technique de l'Institut National des Sciences de l'Univers (INSU)" and the "Centre d'Aviation 
Météorologique de Météo-France" who operated the aircraft and processed the data. B. Bénech, A. Druilhet, F. Saïd and A. Weill contributed to this work by fruitful discussions and valuable comments on the manuscript. This work was financially supported by the "Programme Atmosphère et Océan à Moyenne Echelle de l'INSU/CNRS".

\section{References}

Albrecht, B. A., Bretherton, C. S., Johnson, D., Schubert, W. H., and Frish, A. S.: 1995, 'The Atlantic Stratocumulus Transition Experiment - ASTEX', Bull. Amer. Meteorol. Soc. 76, 889-904.

Betts, A. K., Bretherton, C. S., and Klinker, E.: 1995, 'Relation between Mean Boundary-Layer Structure and Cloudiness at the R/V Valdivia during ASTEX', J. Atmos. Sci. 52, 2752-2762.

Caughey, S. J. and Palmer, S. G.: 1979, 'Some Aspects of Turbulence Structure through the Depth of the Convective Boundary Layer', Quart. J. Roy. Meteorol. Soc. 105, 811-827.

Chou, S. H. and Zimmerman, J.: 1989, 'Bivariate Conditional Sampling of Buoyancy Flux during an Intense Cold-Air Outbreak', Boundary-Layer Meteorol. 46, 93-112.

Deardorff, J. W.: 1970, 'Convective Velocity and Temperature Scales for the Unstable Planetary Boundary Layer and for Rayleigh Convection', J. Atmos. Sci. 27, 1211-1215.

Deardorff, J. W.: 1974, 'Three-Dimensional Numerical Study of Turbulence in an Entraining Mixed Layer', Boundary-Layer Meteorol. 7, 199-226.

Druilhet, A., Frangi, J. P., Guedalia, D., and Fontan, J.: 1983, 'Experimental Studies of the Turbulence Structure Parameters of the Convective Boundary Layer', J. Clim. Appl. Meteorol. 22, 593-608.

Frangi, J. P.: 1979, Contribution to the Study of the Principal Turbulence Characteristics of the Planetary Boundary Layer (in French), Ph.D. Thesis, University of Paris VII, France, 129 pp.

Garratt, J. R.: 1992, The Atmospheric Boundary Layer, Cambridge Atmospheric and Space Science Series, Cambridge University Press, 316 pp.

Greenhut, G. K. and Khalsa, S. J. S.: 1982, 'Updraft and Downdraft Events in the Atmospheric Boundary Layer over the Equatorial Ocean', J. Atmos. Sci. 39, 1803-1818.

Kaimal, J. C., Wyngaard, J. C., Haugen, D. A., Coté, O. R., Izumi, Y., Caughey, S. J., and Readings, C. J.: 1976, 'Turbulence Structure in the Convective Boundary Layer', J. Atmos. Sci. 33, 2152-2168.

Kaimal, J. C. and Finnigan, J. J.: 1994, Atmospheric Boundary Layer Flows. Their Structure and Measurement, Oxford University Press, New York, $289 \mathrm{pp}$.

Khalsa, S. J. S.: 1993, 'Direct Sampling of Entrainment Events in a Marine Stratocumulus Layer', J. Atmos. Sci. 50, 1734-1750.

Lenschow, D. H., Wyngaard, J. C., and Pennel, W. T.: 1980, 'Mean-Field and Second-Moment Budgets in a Baroclinic, Convective Boundary-Layer', J. Atmos. Sci. 37, 1313-1326.

Lenschow, D. H. and Stephens, P. L.: 1980, 'The Role of Thermals in the Convective Boundary Layer', Boundary-Layer Meteorol. 19, 509-532.

Lenschow, D. H. and Stephens, P. L.: 1982, 'Mean Vertical Velocity and Turbulence Intensity Inside and Outside Thermals', Atmos. Environ. 16, 761-764.

Lenschow, D. H. and Stankov, B. B.: 1986, 'Length Scales in the Convective Boundary Layer', $J$. Atmos. Sci. 43, 1198-1209.

Lenschow, D. H., Mann, J., and Kristensen, L.: 1994, 'How Long is Long Enough when Measuring Fluxes and Other Turbulence Statistics?', J. Atmos. Ocean. Tech. 11, 661-673.

Mann, J. and Lenschow, D. H.: 1994, 'Errors in Airborne Flux Measurements', J. Geophys. Res. 99, 14519-14526.

Mahrt, L. and Paumier, J.: 1984, 'Heat Transport in the Atmospheric Boundary Layer', J. Atmos. Sci. 41, 3061-3075.

Nicholls, S.: 1989, 'The Structure of Radiatively Driven Convection in Stratocumulus', Quart. J. Roy. Meteorol. Soc. 115, 487-511.

Randall, D. A., Shao, Q., and Moeng, C.: 1992, 'A Second-Order Bulk Boundary-Layer Model', J. Appl. Meteorol. 49, 1903-1923. 
Réchou, A.: 1995, Turbulence Structure of the Marine Atmospheric Boundary Layer (SOFIA Experiment) (in french), Ph.D. Thesis, University Paul Sabatier, Toulouse, France, 183 pp.

Réchou, A., Durand, P., Druilhet, A., and Bénech, B.: 1995, 'Turbulence Structure of the Boundary Layer Below Marine Clouds in the SOFIA Experiment', Ann. Geophys. 13, 1075-1086.

Shaw, W. J. and Businger, J. A.: 1985, 'Intermittency and the Organization of Turbulence in the Near-Neutral Marine Atmospheric Boundary Layer', J. Atmos. Sci. 28, 918-928.

Weill, A., Klapisz, C., Strauss, B., Baudin, F., Jaupart, C., Van Grunderbeeck, P., and Goutorbe, J. P.: 1980, 'Measuring Heat Flux and Structure Functions of Temperature Fluctuations with an Acoustic Doppler Sodar', J. Appl. Meteorol. 19, 199-205.

Weill, A., Baudin, F., Dupuis, H., Eymard, L., Frangi, J.P., Gérard, E., Durand, P., Bénech, B., Dessens, J., Druilhet, A., Réchou, A., Flamant, P., Seze, G., Pelon, J., Brenguier, J. L., Planton, S., Rolland, J., Leborgne, P., Marsoin, A., Katsaros, K., Queffelou, Y., Tournadre, J., Taylor, P. K., Kent, E., Pascal, R., Schibler, P., Parol, F., Descloitre, J., and Ballois, J. Y.: 1996, 'SOFIA 1992 Experiment during ASTEX', The Global Atmosphere and Ocean System 3, 355-395.

Wyngaard, J. C.: 1983, 'Lectures on the Planetary Boundary Layer', in D. K. Lilly and T. Gal-Chen (eds.), Mesoscale Meteorology - Theories, Observations and Models, pp. 603-650.

Wyngaard, J. C. and Brost, R. A.: 1984, 'Top-Down and Bottom-Up Diffusion of a Scalar in the Convective Boundary Layer', J. Atmos. Sci. 41, 102-112.

Young, G. S.: 1988, 'Turbulence Structure of the Convective Boundary Layer. Part III: TheVertical Velocity Budgets of Thermals and Their Environment', J. Atmos. Sci. 45, 2039-2049. 